Case Reports
in Dermatology

\title{
Anti-MDA5 Antibody-Positive Interstitial Pneumonia with Autoimmune Features Presenting as Amyopathic Hypodermatitic Dermatomyositis: A Case Report
}

\author{
Maria L. Mihailescu ${ }^{a}$ Cuoghi Edens ${ }^{a, b}$ Mark D. Hoffman ${ }^{a, c}$ \\ aUniversity of Chicago, Pritzker School of Medicine, Chicago, IL, USA; bSections of \\ Rheumatology and Pediatric Rheumatology, Departments of Internal Medicine and \\ Pediatrics, University of Chicago Medical Center, Chicago, IL, USA; 'Section of \\ Dermatology, Department of Internal Medicine, University of Chicago Medical Center, \\ Chicago, IL, USA
}

\section{Keywords}

Amyopathic dermatomyositis · Dermatomyositis sine dermatitis · Melanoma differentiation-associated gene $5 \cdot$ Anti-MDA 5 antibody $\cdot$ Autoimmune diseases · Amyopathic hypodermatitic dermatomyositis

\begin{abstract}
Dermatomyositis (DM) and its variant, clinically amyopathic $\mathrm{DM}$, are widely recognized entities. DM sine dermatitis, a variant without skin involvement, is less widely reported. DM with neither muscle nor skin manifestations has not been reported. We herein describe the first account of a patient with a myositis-specific antibody presenting with an array of clinical findings in the absence of both muscle and pathognomonic skin disease. This case report details the multidisciplinary assessment of an anti-melanoma differentiation-associated gene 5 (MDA5) antibody-positive individual with inflammatory polyarthropathy, mucocutaneous capillary changes, and evidence of interstitial lung disease but lacking overt skin and muscle disease. This presentation is paradoxically but appositely deemed to represent a unique form of DM, which may be best described as "amyopathic hypodermatitic dermatomyositis." Early
\end{abstract}




\section{Case Reports in Dermatology}

\begin{tabular}{l|l}
\hline Case Rep Dermatol 2021;13:222-229 \\
\hline DOI: 10.1159/000515245 & $\begin{array}{l}\text { (C) 2021 The Author(s). Published by S. Karger AG, Basel } \\
\text { www.karger.com/cde }\end{array}$ \\
\hline
\end{tabular}

Mihailescu et al.: Anti-MDA5 Antibody-Positive Amyopathic Hypodermatitic Dermatomyositis

recognition and documentation of these cases will help to characterize this variant in the future, determine its frequency, and guide management.

\section{Introduction}

Dermatomyositis (DM) is an autoimmune inflammatory disorder classically having both skin and muscle manifestations. Affected individuals are also at increased risk for interstitial lung disease (ILD) and cancer. Twenty percent of DM patients have absent or minimal muscle disease and are classified as having cutaneous DM sine myositis, also known as clinically amyopathic DM (CADM). Myositis-specific antibody (MSA) testing is implemented to help stratify the clinical course, complications, and treatment outcomes. Some individuals with CADM exhibit MSA that targets melanoma differentiation-associated gene 5 (MDA5) and have a clinical profile associated with unique cutaneous findings (i.e., skin ulceration, palmar papules, oral mucosal pain) and a high incidence of ILD; these patients may also have severe arthritis [1].

DM with muscle inflammation but lacking skin disease - DM sine dermatitis (DMSD) - is more unusual. While a recent report has suggested that nearly $10 \%$ of DM cases may present as DMSD [2], prior to this study only 15 instances of DMSD had been described in paper or poster form [3-11].

We herein describe a patient presenting with a constellation of findings including ILD, inflammatory polyarthropathy, and proximal nail fold and gingival telangiectasias who was found to be anti-MDA5 antibody positive, but who had no clinical or laboratory evidence of myositis and no pathognomonic DM skin changes. The patient's mucocutaneous findings over a 6-month period of follow-up remained limited solely to these fairly characteristic but not DM-specific oral and nail fold changes. We propose that this presentation represents "amyopathic hypodermatitic dermatomyositis" - a presentation of DM with neither clinically evident muscle disease nor overt and pathognomonic skin changes - and which to our knowledge has not been reported previously.

\section{Case Report}

A 19-year-old Caucasian man with no significant past medical history was referred to our dermatology clinic for cutaneous evaluation in the context of presumed anti-MDA5 antibodypositive DM. The patient had initially been seen 1 month prior by a rheumatologist for a 3month history of polyarthralgia and skin changes of the nail folds. He initially described his joints as stiff, swollen, and painful, specifically affecting the toes, ankles, knees, fingers, wrists, and elbows. He had associated morning stiffness lasting $>60 \mathrm{~min}$. The rash was described as redness at the bases of all 10 fingernails. He had been empirically treated with diclofenac 75 $\mathrm{mg}$ twice daily and a methylprednisolone dose pack (24 mg tapered over 6 days) for suspected inflammatory arthritis with improvement. Laboratory explorations found only a low positive anti-MDA5 antibody.

Since symptom onset, he also endorsed fatigue, gingival irritation and bleeding, as well as an unintentional 10-kg weight loss. He denied muscular complaints such as cramping, pain, stiffness, or weakness. He did not experience Raynaud's phenomenon. He reported a 2-year use of an e-cigarette/vaping product with an average use of 1 cartridge per week. He reported

\section{Karger'=}




\section{Case Reports in Dermatology}

Case Rep Dermatol 2021;13:222-229

DOI: $10.1159 / 000515245$

(c) 2021 The Author(s). Published by S. Karger AG, Basel www.karger.com/cde

Mihailescu et al.: Anti-MDA5 Antibody-Positive Amyopathic Hypodermatitic Dermatomyositis

his last use around the time of symptom onset. Cutaneous examination by our dermatology service approximately 4 months after the onset of symptoms showed periungual erythema with capillary loop dilatation (Fig. 1) at the bases of all 10 fingers, and a single small, mildly erythematous, and hyperkeratotic patch on the lateral aspect of the 2nd digit. He never exhibited evidence of a heliotrope or poikilodermatous rash, Gottron's papules or sign, palmar papules, ulcerations, or other hand changes suggestive of "mechanics hands." Subsequent physical examination by our rheumatology service confirmed bilateral synovitis, swelling, and tenderness of the proximal interphalangeal joints of the hands, toes, and elbows; strength was intact. Examination of his oropharynx was notable for gingival telangiectasias (Fig. 2). Prednisone 30 mg daily was started due to clinical concern for an expression of MDA5 antibody-positive DM.

The results of repeat laboratory testing showed an elevated sedimentation rate level of $25 \mathrm{~mm} / \mathrm{h}$ (normal: $0-15$ ) and ferritin at $521 \mathrm{ng} / \mathrm{mL}$ (normal: 20-300). Creatine kinase, aldolase, LDH, and AST were within normal limits and unchanged from previous documentation. Immunologic explorations utilizing a line immunoassay (ARUP Laboratories, Salt Lake City, UT, USA) found "low positive" (no numeric reference value) anti-MDA5 antibodies, which when repeated 2 months later by immunoprecipitation (Oklahoma Medical Research Foundation, Oklahoma City, OK, USA) was reported as "positive." Indirect immunofluorescence found an ANA titer of 1:160 with a speckled pattern. All other autoantibody testing was performed utilizing a line immunoassay, immunoprecipitation, and multiplex bead assays and was negative, including DM-specific autoantibodies (Mi-2, TIF- $\gamma$, NXP2, and SAE1), anti-synthetase syndrome autoantibodies (Jo-1, PL-7, PL-12, EJ, and OJ), an immune-mediated necrotizing myopathy autoantibody (SRP), and other connective tissue disease (CTD)-related (including myositis-associated) autoantibodies (Ro, Ro52, La, anti-Sm, PM/Scl, Scl-70, and ANCA).

Computed tomography of his thorax showed a $17 \times 9 \mathrm{~mm}$ interstitial ground-glass opacity suggestive of ILD. Pulmonary function testing revealed evidence of air trapping with a residual volume of $141 \%$ predicted and a diffusion capacity corrected for an alveolar volume of $80 \%$ predicted. A pulmonary consultant specializing in ILD deemed these lung findings consistent with CTD-associated ILD. A transthoracic echocardiogram was normal. Malignancy work-up, which included testicular ultrasound and CT of the abdomen and pelvis with contrast, was unremarkable.

At this time, there continued to be no evidence of muscle involvement, and skin disease remained limited to the findings previously noted. His arthritis improved on moderate-dose prednisone and he was completely asymptomatic 18 months after initial onset of disease symptoms with plans to transition to a disease-modifying antirheumatic drug.

\section{Discussion and Conclusion}

While DM sine myositis/CADM is a well-recognized and not uncommon subtype of DM, DMSD has not been accorded the same universal inclusion in inflammatory myositis classifications, and fewer than a dozen reports are identified. DMSD has been previously and casually described as cases of DM where the skin findings are "transient or poorly recognized" [12]. More formally, the 2004 ENMC classification criteria for inflammatory myopathies included a category for DMSD, achieving, however, a status of only a "possible" category; elevated CK, EMG or MRI or MSA abnormalities, appropriate muscle biopsy findings, and absence of "rash" are stipulated [13].

\section{Karger'=}




\section{Case Reports in Dermatology}

Case Rep Dermatol 2021;13:222-229

DOI: $10.1159 / 000515245$

(c) 2021 The Author(s). Published by S. Karger AG, Basel www.karger.com/cde

Mihailescu et al.: Anti-MDA5 Antibody-Positive Amyopathic Hypodermatitic Dermatomyositis

Historically, DM classification was satisfied based on criteria proposed by Bohan and Peter in 1975; their program does not recognize cases of DM without some degree of both muscle and skin involvement [14]. Neither the ENMC nor the Bohan and Peter criteria have ever been validated. In 2017, the European League against Rheumatism/American College of Rheumatology (EULAR/ACR) released the first validated classification criteria for DM/polymyositis (PM) that captures a wider array of entities on its myositis spectrum [15]. Still, these guidelines remain restricted to cases with pathognomonic skin lesions (heliotrope rash, Gottron's papules, and/or Gottron's sign) and also fail to recognize myositis-specific antibodies such as anti-MDA5 [16], excluding our patient from classification. While our patient's nailfold capillary and gingival changes suggest DM, neither feature is considered pathognomonic for DM or CADM and would thus not suffice for a diagnosis. We will refer to these changes as "hypodermatitic" to reflect these clinically important but diagnostically insufficient features. Also, as myositis-specific antibodies are becoming increasingly recognized as important diagnostic tools, experts recommend they be adopted into any future guidelines for characterizing DM [17]. One such retrospective analysis by Allenbach et al. [18] aimed to characterize the anti-MDA5+ DM phenotype into three subgroups based on selected concomitant variables such as Raynaud's phenomenon, arthritis/arthralgias, and gender. Our patient would be segregated into the prognostically favorable "cluster 2," which is characterized by less frequent skin lesions, a lower tendency to develop muscular manifestations, an intermediate risk of ILD, and higher rates of arthralgia.

As our patient lacked clinical muscle weakness, had normal muscle enzymes, and exhibited a normal muscular examination result as evaluated by two experienced rheumatologists, no muscle biopsy, further imaging, or electromyography was pursued. However, had our patient been subjected to such evaluations, it is possible that he may have had subclinical evidence of muscle involvement and would thus be characterized as having "hypomyopathic" rather than "amyopathic" disease [19].

Although two series of DM patients have found the prevalence of DMSD to be between 8 and $15 \%[2,3]$, the true frequency of occurrence of this entity within DM cohorts is unknown. Cases such as ours deemed to represent DM but having neither muscle nor pathognomonic skin manifestations are without published precedence, and might seem nonsensical, although counterparts do exist (e.g., systemic sclerosis sine scleroderma). It has been suggested that vascular features such as abnormal nailfold capillaroscopy results - as found in our patient are of paramount importance in recognizing DMSD [20]. Moreover, a recent Delphi exercise has generated a potential list of classification criteria for DM and emphasized the critical importance of signals such as MSAs and ILD. Both were included (with nailfold capillary loops) in the final consensus item pool that was produced (neither gingival telangiectasias nor joint disease were proposed for consideration) [21].

Recently, González-Moreno et al. [22] described a case of anti-MDA5 antibody-associated, rapidly progressive ILD in a patient with arthritis but no other clinical signs of muscle or skin disease from the time of diagnosis through 10 months of follow-up. A case of an anti-MDA5 antibody-positive patient who developed rapidly progressive ILD without cutaneous manifestations initially, but after 1 month showed Gottron's sign and mechanic's hands, has been reported [23]. Ahsan and Erum [24] recently reported a case of DM without anti-MDA5 positivity that presented with intermittent urticarial rashes and diffuse arthralgias for 1 year; interestingly, the patient only developed the characteristic cutaneous and then muscular symptoms 3-4 years later. It is unclear if such cases convert over time into one of the more recognizable entities DM, CADM, or DMSD. Alternatively, ours and perhaps other cases may have

\section{Karger'=}




\section{Case Reports in Dermatology}

\begin{tabular}{l|l}
\hline Case Rep Dermatol 2021;13:222-229 \\
\hline DOI: 10.1159/000515245 & $\begin{array}{l}\text { @ 2021 The Author(s). Published by S. Karger AG, Basel } \\
\text { www.karger.com/cde }\end{array}$ \\
\hline
\end{tabular}

Mihailescu et al.: Anti-MDA5 Antibody-Positive Amyopathic Hypodermatitic Dermatomyositis

had authentic but extremely subtle skin manifestations that eluded recognition. It is also conceivable that some such cases had "invisible" skin disease, whereby distinctive histologic interface changes might have been disclosed by "blind" biopsies. Lastly, underreporting of similar cases may be relevant and a product of medical advancement - the anti-MDA5 antibody was not recognized until the 21st century [25], and commercial testing has been available only within the decade.

Anti-MDA5 has been considered to be highly specific for DM. In two studies assessing the anti-MDA5 status in both DM and PM, anti-MDA5 was only detected in the DM cases, and was found in $0 \%$ of the PM patients [26, 27]. Sato et al. [28] tested a cohort of 255 patients with a variety of CTDs including DM, PM, systemic lupus erythematosus, mixed CTD, systemic sclerosis, and Sjögren's syndrome, in addition to patients with idiopathic pulmonary fibrosis and normal human controls; anti-MDA5 was detected in 53\% of the CADM subset but was not present in the others.

Patients having ILD with features suggestive but not diagnostic of a specific CTD do occur as above and have been designated by the European Respiratory Society/American Thoracic Society as interstitial pneumonia with autoimmune features (IPAF) [29]. Our patient appears to fit this category, having ILD plus arthritis and three features characteristic of DM being antiMDA5 antibody positive and exhibiting periungual and gingival capillary dilatation; thus, "anti-MDA5+ IPAF" would seem to be a suitable alternative designation. From an operational standpoint and for purposes of ongoing dermatologic, rheumatologic, pulmonary, and potentially oncologic surveillance, characterization as a DM variant does indeed also appear appropriate. A 2020 study of patients with CTD-related (including DM) ILD and IPAF found antiMDA5 antibodies present in 44\% of CTD-related cases (the specific CTD type[s] were not specified), but also in $16 \%$ of the IPAF cases, suggesting that anti-MDA5 is not uncommonly associated with autoimmune presentations with atypical or only inconclusive features, such as ours [30].

Of additional interest is our patient's history of vaping prior to the onset of his anti-MDA5positive inflammatory disorder. E-cigarette use or vaping is associated with lung disease (ecigarette, or vaping, product use-associated lung injury or EVALI) and has been linked to vitamin E acetate [31]. E-cigarette use has also been linked with augmented NETosis (neutrophil extracellular traps [NETs], consisting of filaments and granule proteins, and linked with CTDs) [32]; moreover, NETosis has been associated with anti-MDA5-positive DM [33]. The possible pathogenic role for vaping in our patient is another intriguing but uncertain facet of the presentation.

\section{Statement of Ethics}

This research complies with the guidelines for human studies and was conducted ethically in accordance with the World Medical Association Declaration of Helsinki. Written informed consent was obtained from the patient for publication of this case report and any accompanying images.

\section{Conflict of Interest Statement}

The authors have no conflicts of interest to declare.

\section{Karger'=}




\section{Case Reports in Dermatology}

\section{Funding Sources}

The authors received no funding for this work.

\section{Author Contributions}

All authors meet the criteria for authorship as stated in the 2013 guidelines of the International Committee of Medical Journal Editors. M.L.M. is responsible for conceptualization and design of the work, data analysis and interpretation, and drafting and revision of the work. M.L.M. has approved of the version to be published and agrees to be held accountable for all aspects of the work. M.D.H. is responsible for conceptualization and design of the work, data acquisition, analysis and interpretation, and drafting and revision of the content. M.D.H. has approved of the version to be published and agrees to be held accountable for all aspects of the work. C.E. is responsible for conceptualization and design of the work, data acquisition analysis and interpretation, and drafting and revision of the content. C.E. has approved of the version to be published and agrees to be held accountable for all aspects of the work.

\section{References}

1 Fiorentino D, Chung L, Zwerner J, Rosen A, Casciola-Rosen L. The mucocutaneous and systemic phenotype of dermatomyositis patients with antibodies to MDA5 (CADM-140): a retrospective study. J Am Acad Dermatol. 2011 Jul;65(1):25-34.

2 Inoue M, Tanboon J, Hirakawa S, Komaki H, Fukushima T, Awano H, et al. Association of Dermatomyositis sine Dermatitis with Anti-Nuclear Matrix Protein 2 Autoantibodies. JAMA Neurol. 2020 Jul;77(7):872-7.

3 Karri SB, Kannan MA, Rajashekhar L, Uppin MS, Challa S. Clinico pathological study of adult dermatomyositis: importance of muscle histology in the diagnosis. Ann Indian Acad Neurol. 2015 Apr-Jun;18(2):194-9.

4 Park JS, Park JY. Dermatomyositis sine dermatitis, a rare phenotype of idiopathic inflammatory myopathy. Yeungnam Univ J Med. 2017 Jun;34(1):137-9.

5 Vembu K, Lavingia J, Mankad J. Dermatomyositis with absent skin findings. 2012. DOI: https://doi.org/10.13140/2.1.2024.3204.

6 Papakonstantinou E, Kapp A, Raap U. A mild form of dermatomyositis as a prodromal sign of lung adenocarcinoma: a case report. J Med Case Reports. 2016 Feb;10(1):34.

7 Nilipour Y, Ghiasi M, Rohani M, Omrani F. Juvenile dermatomyositis without skin lesions. Iran J Neurol. 2015 Jul;14(3):171-3.

8 Choi YS, Ryu KH, Kwon GY, Jeon SS, Choi HY, Lee HM. Ureteral cancer associated with dermatomyositis. Int J Urol. 2006 Apr;13(4):442-4.

9 Szwebel TA, Perrot S, Kierzek G, Maisonobe T, Tigaud JM, Le Jeunne C, et al. Paraneoplasic dermatomyositis sine dermatitis associated with a tumor of the renal excretion system. J Clin Neuromuscul Dis. 2008 Sep;10(1):35-6.

10 George MD, Lahouti AH, Christopher-Stine L. An atypical case of dermatomyositis associated with chromophobe renal cell carcinoma. BMJ Case Rep. 2016 Jan;2016:bcr2015212387.

11 Kyaw H, Shaikh AZ, Ayala-Rodriguez C, Deepika M. Paraneoplastic cardiac involvement in renal cell carcinoma with dermatomyositis sine dermatitis. Ochsner J. 2017;17(4):421-5.

12 Dalakas MC, Hohlfeld R. Polymyositis and dermatomyositis. Lancet. 2003 Sep;362(9388):971-82.

13 Hoogendijk JE, Amato AA, Lecky BR, Choy EH, Lundberg IE, Rose MR, et al. 119th ENMC international workshop: trial design in adult idiopathic inflammatory myopathies, with the exception of inclusion body myositis, 10-12 October 2003, Naarden, The Netherlands. Neuromuscul Disord. 2004 May;14(5):337-45.

14 Bohan A, Peter JB. Polymyositis and dermatomyositis (first of two parts). N Engl J Med. 1975 Feb;292(7):344-7.

15 Lundberg IE, Tjärnlund A, Bottai M, Werth VP, Pilkington C, Visser M, et al.; International Myositis Classification Criteria Project consortium, The Euromyositis register and The Juvenile Dermatomyositis Cohort Biomarker Study and Repository (JDRG) (UK and Ireland). 2017 European League Against 


\section{Case Reports in Dermatology}

Case Rep Dermatol 2021;13:222-229

DOI: $10.1159 / 000515245$

(c) 2021 The Author(s). Published by S. Karger AG, Basel www.karger.com/cde

Mihailescu et al.: Anti-MDA5 Antibody-Positive Amyopathic Hypodermatitic Dermatomyositis

Rheumatism/American College of Rheumatology classification criteria for adult and juvenile idiopathic inflammatory myopathies and their major subgroups. Ann Rheum Dis. 2017 Dec;76(12):1955-64.

16 Satoh M, Tanaka S, Ceribelli A, Calise SJ, Chan EK. A Comprehensive Overview on Myositis-Specific Antibodies: New and Old Biomarkers in Idiopathic Inflammatory Myopathy. Clin Rev Allergy Immunol. 2017 Feb;52(1):1-19.

17 Gono T, Kuwana M. Current understanding and recent advances in myositis-specific and -associated autoantibodies detected in patients with dermatomyositis. Expert Rev Clin Immunol. 2020 Jan;16(1):79-89.

18 Allenbach Y, Uzunhan Y, Toquet S, Leroux G, Gallay L, Marquet A, et al.; French Myositis Network. Different phenotypes in dermatomyositis associated with anti-MDA5 antibody: study of 121 cases. Neurology. 2020 Jul;95(1):e70-8.

19 Sontheimer RD. Would a new name hasten the acceptance of amyopathic dermatomyositis (dermatomyositis sine myositis) as a distinctive subset within the idiopathic inflammatory dermatomyopathies spectrum of clinical illness? J Am Acad Dermatol. 2002 Apr;46(4):626-36.

20 Leteurtre E, Hachulla E, Janin A, Hatron PY, Brouillard M, Devulder B. Vascular manifestations of dermatomyositis and polymyositis. Clinical, capillaroscopic and histological aspects [in French]. Rev Med Interne. 1994;15(12):800-7.

21 Concha JS, Pena S, Gaffney RG, Patel B, Tarazi M, Kushner CJ, et al.; Skin Myositis Delphi Group. Developing classification criteria for skin-predominant dermatomyositis: the Delphi process. Br J Dermatol. 2020 Feb;182(2):410-7.

22 González-Moreno J, Raya-Cruz M, Losada-Lopez I, Cacheda AP, Oliver C, Colom B. Rapidly progressive interstitial lung disease due to anti-MDA5 antibodies without skin involvement: a case report and literature review. Rheumatol Int. 2018 Jul;38(7):1293-6.

23 Tamai K, Tachikawa R, Otsuka K, Ueda H, Hosono Y, Tomii K. Early pulmonary involvement of anti-CADM140 autoantibody-positive rapidly progressive interstitial lung disease preceding typical cutaneous symptoms. Intern Med. 2014;53(21):2515-9.

24 Ahsan T, Erum U. An elusive case of dermatomyositis. J Pak Med Assoc. 2017 Nov;67(11):1756-8.

25 Sato S, Hoshino K, Satoh T, Fujita T, Kawakami Y, Fujita T, et al. RNA helicase encoded by melanoma differentiation-associated gene 5 is a major autoantigen in patients with clinically amyopathic dermatomyositis: association with rapidly progressive interstitial lung disease. Arthritis Rheum. 2009 Jul;60(7):2193-200.

26 Ceribelli A, Fredi M, Taraborelli M, Cavazzana I, Tincani A, Selmi C, et al. Prevalence and clinical significance of anti-MDA5 antibodies in European patients with polymyositis/dermatomyositis. Clin Exp Rheumatol. 2014 Nov-Dec;32(6):891-7.

27 Chen Z, Cao M, Plana MN, Liang J, Cai H, Kuwana M, et al. Utility of anti-melanoma differentiation-associated gene 5 antibody measurement in identifying patients with dermatomyositis and a high risk for developing rapidly progressive interstitial lung disease: a review of the literature and a meta-analysis. Arthritis Care Res (Hoboken). 2013 Aug;65(8):1316-24.

28 Sato S, Hirakata M, Kuwana M, Suwa A, Inada S, Mimori T, et al. Autoantibodies to a 140-kD polypeptide, CADM-140, in Japanese patients with clinically amyopathic dermatomyositis. Arthritis Rheum. 2005 May;52(5):1571-6.

29 Fischer A, Antoniou KM, Brown KK, Cadranel J, Corte TJ, du Bois RM, et al.; "ERS/ATS Task Force on Undifferentiated Forms of CTD-ILD". An official European Respiratory Society/American Thoracic Society research statement: interstitial pneumonia with autoimmune features. Eur Respir J. 2015 Oct;46(4):976-87.

30 Tian M, Huang W, Ren F, Luo L, Zhou J, Huang D, et al. Comparative analysis of connective tissue diseaseassociated interstitial lung disease and interstitial pneumonia with autoimmune features. Clin Rheumatol. 2020 Feb;39(2):575-83.

31 Blount BC, Karwowski MP, Shields PG, Morel-Espinosa M, Valentin-Blasini L, Gardner M, et al.; Lung Injury Response Laboratory Working Group. Vitamin E Acetate in Bronchoalveolar-Lavage Fluid Associated with EVALI. N Engl J Med. 2020 Feb;382(8):697-705.

32 Reidel B, Radicioni G, Clapp PW, Ford AA, Abdelwahab S, Rebuli ME, et al. E-Cigarette Use Causes a Unique Innate Immune Response in the Lung, Involving Increased Neutrophilic Activation and Altered Mucin Secretion. Am J Respir Crit Care Med. 2018 Feb;197(4):492-501.

33 Peng Y, Zhang S, Zhao Y, Liu Y, Yan B. Neutrophil extracellular traps may contribute to interstitial lung disease associated with anti-MDA5 autoantibody positive dermatomyositis. Clin Rheumatol. 2018 Jan;37(1):107-15.

\section{Karger'=}




\section{Case Reports in Dermatology}

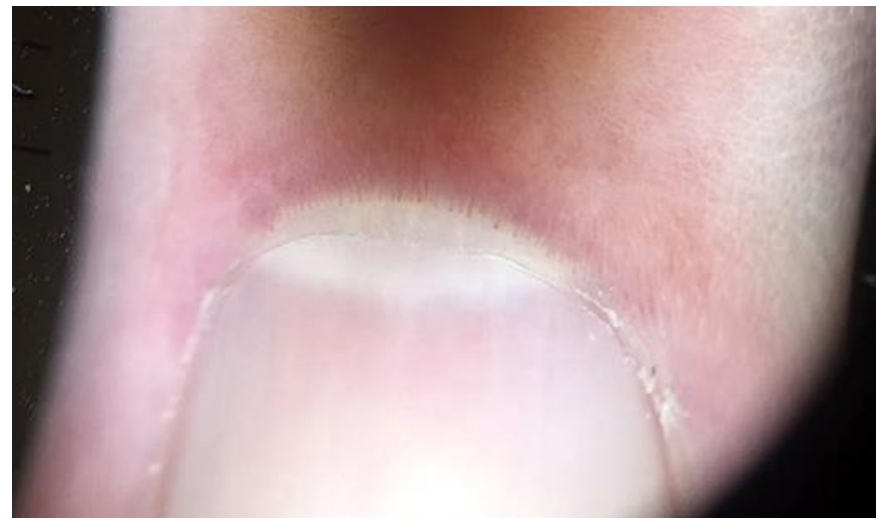

Fig. 1. Proximal nailfold demonstrating several enlarged capillaries.

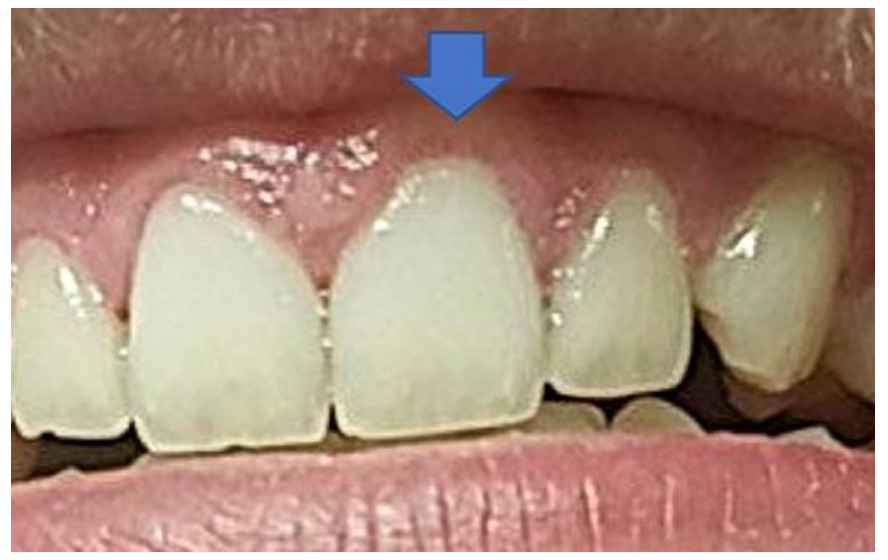

Fig. 2. Telangiectasias along the marginal gingivae (arrow) and interdental papillae. 